\title{
Piedad de la Cierva in the Instituto de Óptica “Daza de Valdés" and her contribution to the optics research
}

\section{Piedad de la Cierva Viudes en el Instituto de Óptica "Daza de Valdés" y su contribución en la investigación óptica}

\author{
Giovanni Zen \\ Universidad Camilo José Cela, Madrid, España \\ Email: zengiov@gmail.com \\ Received: $12 / 12 / 2017 \quad$ Accepted: 15/10/2018 \\ DOI: $10.7149 /$ OPA.51.4.50022
}

\begin{abstract}
:
Piedad de la Cierva Viudes (Murcia 1913-Madrid 2007) has been one among the female pioneers in science whose name, unfortunately, does not appear in history books. Nevertheless, her contribution to Optics and Chemistry is highly relevant in Spain between the Second Spanish Republic and the first years of Franco's regime. In this article I would like to highlight her work at the Instituto de Óptica "Daza de Valdés", recently created in those years and specifically her research on glass and on anti-reflective sheets.
\end{abstract}

Key words: Piedad de la Cierva Viudes, Instituto de Óptica, CSIC, gender, pioneers

\section{RESUMEN:}

Piedad de la Cierva Viudes (Murcia 1913-Madrid 2007) ha sido una de las mujeres pioneras en las ciencias cuyo nombre desgraciadamente no aparece todavía en los libros de historia. Su contribución en Óptica y en Química fue, sin embargo, de elevado valor e importancia en España entre la II República y los primeros años del Franquismo. En este artículo quiero destacar su aportación a la investigación en el, por aquel entonces, recién creado Instituto de Óptica Daza de Valdés y, en particular, acerca de sus estudios sobre el vidrio, las láminas antirreflectoras y los cristales.

Palabras clave: Piedad de la Cierva, Instituto de Óptica, CSIC, género, pioneras

\section{REFERENCES AND LINKS / REFERENCIAS Y ENLACES}

[1] En la Universidad de Murcia no había mujeres en el año en el que se matriculó Piedad. Véase datos INE http://www.ine.es/inebaseweb/pdfDispacher.do?td=150210\&ext=.pdf (consultado el 21/03/2017).

[2] M. Montero, La conquista del espacio público. Mujeres en la universidad española (1910-1936). Madrid, Minerva Ediciones. (2009), pág. 112

[3] J. Cuesta, M.J. Turrión, R.M. Merino, La residencia de señoritas y otras redes culturales femeninas. Salamanca, Ediciones Universidad de Salamanca Fundación Ortega y Gasset-Gregorio Marañón, (2015), pág. 46.

[4] http://www.ine.es/inebaseweb/pdfDispacher.do?td=150210\&ext=.pdf $\quad$ (recuperado el 16/07/2018) para el año 1928-29

[5] En el Archivo Niels Bohr se conserva testigo del trabajo de Piedad en Copenhague con von Hevesy

[6] Laureano López Rodó (Barcelona 1920-Madrid 2000). Político, jurista, catedrático y abogado. Ministro de Asuntos Exteriores 1973-1974.

[7] Archivo Laureano López Rodó. F.005. Caja 4. Universidad de Navarra 
[8] "La organización del Consejo tiene posibilidades de aprovechamiento de la mayor trascendencia para la vida científica, docente y económica del país, y hay que superar esa insana tendencia nativa a la novedad, en virtud de la cual se crean Instituciones importantes; éstas cuentan con medios iniciales que permiten su desarrollo y, cuando al paso de los años puedes ser más eficaces y realizar trabajos más importantes y continuados, al perder el valor de la novedad se limitan sus medios y corren el grave riesgo de perder ímpetu y vigor." Ref. Archivo Laureano López Rodó. F.005. Caja 4. Universidad de Navarra.

[9] Web del IO: http://www.io.csic.es/

[10] 1907-1983. IX marqués de Hermosilla, Contralmirante-ingeniero de la Armada, fue un físico español especializado en el campo de la óptica. Otero realizó importantes estudios en óptica geométrica, física, fisiología y energía nuclear. Por otro lado, Otero puede considerarse como el padre de la energía nuclear en España ya que fue presidente de la Junta de Energía Nuclear desde 1958 hasta 1974. Durante este periodo se creó el primer reactor español, el reactor de la Moncloa (1958) y en 1969 la primera central nuclear española, la José Cabrera. En 1965 fue nombrado presidente de la Sociedad Europea de Energía Atómica y en el 1968 presidente de la Oficina Internacional de Pesos y Medidas. En 1968 fue nombrado gobernador del Organismo Internacional de Energía Atómica (OIEA).

[11] I. Alva Rodríguez, "Piedad de la Cierva: una sorprendente trayectoria profesional durante la segunda república y el franquismo". Arbor, 192 (779): a322, (2016).

[12] A. Romero de Pablos, "Mujeres científicas en la dictadura de Franco. Trayectorias investigadoras de Piedad de la Cierva y María Aránzazu Vigón", Arenal 24:2 (2017), p. 330

[13] Biblioteca del IO, AME/IO/0002/01 Memorias. 1946

[14] Biblioteca del IO, AME/IO/0002/01 Memorias. 1948

[15] P. de la Cierva, Ensayos de fabricación de vidrio óptico, Madrid, CSIC. Patronato "Juan de la Cierva" de Investigación técnica, (1955).

[16] Documento del departamento de inmigración de Estados Unidos en el que se testifica la llegada de Piedad a Nueva York el 22 de junio de 1948 . En https://familysearch.org/ark:/61903/1:1:QVP8-DQQ3 (recuperado el 25/04/2016). "New York, New York Passenger and Crew Lists, 1909, 1925-1957," database with images, FamilySearch (https://familysearch.org/ark:/61903/1:1:QVP8-DQQ3 : accessed 25 April 2016), Piedad De La Cierva, 1948; citing Immigration, New York, United States, NARA microfilm publication T715 (Washington, D.C.: National Archives and Records Administration, n.d.).

[17] The American Ceramic Society Bulletin, vol. 42, núm. 6 de junio de 1963.

[18] AME/I0/0002/04 Memorias. 1949

\section{Introducción}

Hay pocas noticias sobre la infancia de Piedad de la Cierva. Sabemos que entró con nueve años (el 1 de junio de 1923) en el Instituto General y Técnico de Murcia (hoy IES Alfonso X El Sabio). La pequeña Piedad mostró enseguida especial interés en las asignaturas científicas y sacó notas muy altas en geometría, aritmética, dibujo técnico y álgebra. Piedad cursaba la modalidad "no oficial", es decir, tenía que prepararse en casa, estudiar con un tutor para luego presentarse a los exámenes.

Después de estudiar durante un año en la Universidad de Murcia [1], facultad de Ciencias, Piedad de la Cierva se mudó a Valencia en 1928 donde se licenció en Ciencias Químicas en 1932 (con premio extraordinario a los 19 años) con un trabajo sobre hierros y aceros. El ambiente de su ciudad natal le resultó pronto asfixiante y académicamente poco estimulante. Como recoge Mercedes Montero [2] en la Laguna, Murcia e incluso Oviedo, la presencia femenina era meramente testimonial. Por esta razón el valor del recorrido académico y laboral de Piedad de la Cierva es altísimo y se la puede considerar verdaderamente una pionera si consideramos el ambiente y la zona geográfica en la que se formó.

Doctora en Ciencias Químicas por la Universidad Central de Madrid en 1934, estudió en Copenhague (Instituto Niels Bohr, 1935, con solo 21 años), donde conoció y mantuvo una estrecha relación profesional y personal con varias importantes personalidades científicas de la época: Lise Meitner y 
Hermann Staudinger (premio Nobel), George von Hevesy (premio Nobel) y fue huésped de la familia Noddack (dos candidatos al Nobel).

Piedad sigue siendo una figura desconocida a pesar de su importante trayectoria investigativa y puede sin duda incluirse en un grupo de científicas que abrieron paso a la inserción de las mujeres en el mundo de las ciencias. La podemos incluir entre las importantes científicas que solo unos años antes obtuvieron un gran prestigio y considerarla heredera de Jenara Vicenta Arnal Yarza (primera doctora en Ciencias Químicas en España), Ángela García de la Puerta (primera catedrática de Física y Química), María Antonia Zarroquino y Dorotea Barnés González.

Su pasión por las ciencias, su recorrido académico y los trabajos realizados sobre la radiación hicieron que José María Otero Navascués contactara con ella en 1946 para crear una Sección de Óptica, dentro del Instituto de Física Alonso de Santa Cruz, y que trabajara para la Marina creando instrumentos ópticos. Es así que Piedad de la Cierva, doctora en Ciencias Químicas, se fue poco a poco especializando en Óptica.

\section{Mujeres y Universidad}

A pesar de que la ley que permitió a las mujeres acceder a los estudios superiores se promulgó en 1910, esto no significó que la realidad se ajustara a la legislación y el debate sobre la inserción de las alumnas en el mundo universitario seguía vivo en la sociedad. Desde la promulgación de la ley, se aprecia cómo la carrera más elegida por las españolas en el primer tercio del siglo XX fue Farmacia [3] y las primeras inscritas se matriculan, curiosamente, en facultades científicas, socialmente consideradas no aptas para las mujeres. Las letras y el magisterio eran seguramente más indicadas para ellas en la sociedad española. Aun así, sólo las mujeres pertenecientes a las élites intelectuales o las hijas de funcionarios podían inscribirse en la universidad. Cabe mencionar que la clase media en España era entonces bastante escasa.

Al comienzo y animada por su padre, Juan de la Cierva López, abogado, Piedad optó por la universidad de su ciudad natal, Murcia, donde cursó un año en régimen de enseñanza "no oficial". Es fácil concluir que este tipo de enseñanza y en una ciudad periférica como la Murcia del siglo XX, era la más indicada para las mujeres. De hecho, en el año académico 1928-29, ninguna mujer aparece matriculada en la Universidad de Murcia [4].

Cansada por el ambiente cerrado de esta universidad, Piedad decidió entonces hacer un traslado de matrícula a la Universidad de Valencia en 1929.

\section{La discriminación de género. Las oposiciones a cátedra de 1940}

A lo largo de su vida, Piedad sufrió, por el mero hecho de ser mujer, una discriminación muy dura en el mundo académico. Al terminar la Guerra Civil el nuevo gobierno llevó a cabo una profunda reestructuración de la enseñanza en todos sus niveles educativos, desde la escuela primaria hasta la universidad. Por todo ello, enseguida se empezaron a convocar oposiciones para cubrir las plazas vacantes en las diferentes facultades del país. Así a principios de 1941 se anunciaron tres cátedras de Química-Física para las universidades de Madrid, Sevilla y Murcia. Aún no había catedráticos de esta materia pues era una carrera universitaria de reciente creación.

El padre de Piedad quería que optase a la de Murcia porque aún no había mujeres en dicha universidad y le hacía especial ilusión que su hija fuera la primera. A ella, en principio, no, pero fue tanta la presión paterna que al final se decidió a prepararse para el examen.

El Tribunal que juzgaría a los opositores estaba formado por don José Casares Gil, decano de la Facultad de Farmacia de la Universidad de Madrid y Ángel Santos, catedráticos de Farmacia, don Antonio Ríos, de Electroquímica, Alfredo Quijano, ingeniero de Armas Navales y don Antonio Ipiens, que había sido profesor de Piedad en Valencia.

Se presentaron Piedad de la Cierva Viudes, Julián Rodríguez Velasco, María Teresa Salazar Bermúdez, José María González Barredo y Octavio Rafael Foz Gazulla. En las votaciones resultó elegido en primer lugar Octavio Rafael Foz Gazulla, que optó por la cátedra de Valencia, en segundo lugar, quedó Julián 
Rodríguez Velasco que escogió la de Sevilla. Para la tercera cátedra cuatro miembros del tribunal votaron por la no provisión, quedando desierta la de Murcia.

Piedad de la Cierva y María Teresa Salazar, a pesar de unos currículos claramente superiores a los de los otros candidatos, no llegaron nunca a obtener la cátedra, por su vinculación antes de la guerra con el Instituto Nacional de Física y Química de la Junta para la Ampliación de Estudios, por sus maestros Julio Palacios y Enrique Moles, y por su condición de mujeres, a pesar de los impecables avales políticos que presentaron: la primera había sido dirigente de la Sección Femenina de Falange durante la Guerra Civil en Sevilla y la segunda era monja.

Los informes de los miembros del tribunal sobre Piedad de la Cierva Viudes coincidían en líneas similares con la opinión expresada por José Casares Gil, presidente del tribunal:

"Esta opositora presenta una serie de trabajos efectuados en colaboración con los señores Palacios, Rivori y prof. Hevesy de Copenhague"; mientras Ángel Santos Ruiz cuestionó la colaboración con destacados físicos como Julio Palacios y George Hevesy [5], una autoridad mundial sobre las propiedades químicas de las sustancias a través de los isótopos, por los que recibió el premio Nobel de Química en1943. Se le descalificaba porque "ninguno de los trabajos presentados es de iniciativa propia del opositor y todos ellos deben ser considerados como llevados a cabo en colaboración y por iniciativa ajena".

\section{La creación del CSIC}

En 1939 la situación científica en España se encontraba en grave crisis, pero el nuevo gobierno tuvo gran interés en crear el Consejo Superior de Investigaciones Científicas: tanto para sustituir las obras de la República, la Junta para la Ampliación de Estudios y borrar así todo rastro de su actividad, como para crear una cultura científica española más organizada. En el Archivo Laureano López Rodó [6], que se encuentra en la Universidad de Navarra, se encuentran documentos de la época que explican la voluntad de crear una institución científica nacional que diera prestigio a España sobre todo a nivel internacional:

"El desarrollo de la investigación es trascendental en la ideología del país [...] En 1939, pocos meses después de la terminación de la guerra, el Caudillo creó el Consejo Superior de Investigaciones Científicas cuya labor ha superado, de modo decisivo, todos los intentos previos de desarrollo de la investigación científica y ha alcanzado un reconocimiento incomparable" [7].

En su visión, la responsabilidad del CSIC es la de impulsar la ciencia tanto creando centros propios como vinculándose los existentes para que la investigación sea "total" y organizada englobando representaciones de Universidades, Escuelas Técnicas Superiores, Reales Academias, Ciencias Sagradas, Militares, Instituciones privadas [7] en un conjunto científico bien estructurado.

El CSIC es una estructura muy ramificada que financia y asiste otros centros de investigación sin que el gobierno intervenga. Sin embargo, siempre según López Rodó, España tiene una tendencia peligrosa que consiste en abrazar todo lo nuevo y lo novedoso, pero cuando éste pierde el interés, cae en el olvido y nadie ya se ocupa de él [8]. Así la creación de un órgano superior, nacional, es necesaria y goza de una larga vida gracias a la creación de una Fundación Nacional de Investigación que se ocuparía del aspecto económico (mayor obstáculo a la supervivencia del CSIC). La fundación representaría el poder económico de la investigación que juzgara directamente sobre los medios de que ésta dispone, sobre su distribución, sobre la sistematización de los gastos[...] [7].

\section{La creación del Instituto de Óptica "Daza de Valdés"}

El IO (Instituto de Óptica del CSIC) sigue existiendo hoy en día y goza de un gran prestigio internacional. En la página web del Instituto podemos leer:

"El Instituto de Óptica pertenece a la red de centros de Investigación del CSIC. Sus objetivos científicos abarcan todos los aspectos de la óptica y la fotónica, con énfasis en áreas como la Óptica 
Fisiológica, el Procesado de Imágenes, la Metrología Óptica, la Interacción Láser-Materia, la Dinámica No-lineal de Sistemas Ópticos, la Nanofotónica y las Comunicaciones Ópticas" [9].

Fue creado en 1946 por el Profesor José María Otero Navascués [10], entonces jefe de la Sección de Óptica del Instituto de Física "Alonso de Santa Cruz" (Instituto Rockefeller):

"El Instituto se desplazó en 1950 a su ubicación actual, un nuevo edificio, también dentro del campus, diseñado por el arquitecto Fisac. La investigación en el Instituto de Óptica se centraba originalmente en dos áreas principales: visión y espectroscopia, contando en ambas con investigadores de elevado prestigio internacional, cuya actividad se expandió pronto hacia la óptica fisiológica, la exploración ocular, el diseño de sistemas ópticos, la fotografía, la espectroscopia infrarroja y la óptica física; a las que se añadirían en una etapa posterior la microscopía electrónica, el estudio de espectros atómicos, el estudio de materiales luminiscentes, la colorimetría y la fotometría. En 1956 el instituto fue reasignado al Patronato Juan de la Cierva, organismo del CSIC dedicado al desarrollo tecnológico.

En los años siguientes, el Instituto de Óptica, uno de los más antiguos del CSIC, continuó promoviendo la investigación de calidad en óptica, con frecuentes visitas de prestigiosos profesores internacionales, publicaciones en las revistas internacionales de mayor prestigio e importantes colaboraciones internacionales (desde los años 40 y 50), así como desarrollando programas de formación (fundando la primera Escuela de Óptica y Optometría), de transferencia tecnológica (fundando en los años 50 la Empresa Nacional de Óptica) y la promoción de la óptica en la sociedad (fundando la Sociedad Española de Óptica y su revista "Óptica Pura y Aplicada" en los años 60)"[9].

\section{Piedad de la Cierva en el Instituto de Óptica “Daza de Valdés” (1945)}

En la Biblioteca del Instituto de Óptica encontramos una serie de memorias que testifican el gran trabajo que sus científicos, los mejores de la época, desarrollaron en los laboratorios y lo que publicaron. Piedad de la Cierva formó parte del primer núcleo de científicos, seleccionados por Otero Navascués que, durante 5 años, e ininterrumpidamente, trabajó ocupándose de preparar espejos de aluminio por evaporación al vacío, una técnica necesaria para la fabricación de prismáticos [11]. Piedad no sólo formó parte de este conjunto de científicos que empezaron con Navascués, sino que:

"Piedad de la Cierva recibió el encargo del ingeniero de la Armada José María Otero Navascués, personaje clave en la política científica - sobre todo la nuclear- del franquismo, de organizar un laboratorio para construir vidrio óptico en el Laboratorio y Taller del Estado Mayor de la Armada (LTIEMA)." [12]. Cuando Piedad llegó al LTIEMA ya funcionaba el taller mecánico y el taller de Optica y ella sería la encargada de instalar el laboratorio de química donde tuvo que realizar los trabajos más variados.

Con Francisco Castro, químico, como ayudante y una mujer, Julita, como auxiliar de laboratorio, montaron un aparato de evaporación en vacío, para lo que le sirvieron mucho las investigaciones llevadas a cabo en Copenhague en el Instituto creado por Niels Bohr donde Piedad había trabajado en 1935, y consiguieron la preparación de espejos de aluminio para los prismáticos. Muchos personajes de la Marina visitaban el laboratorio cada día para comprobar los avances científicos que iban consiguiendo aquellos jóvenes científicos.

Mientras en España se encontraban en la posguerra, el resto de Europa estaba metido en aquellos momentos en plena guerra mundial. Otero había estado en Alemania y, por aquel entonces, en 1941, la Marina alemana estaba obteniendo grandes éxitos pues los investigadores alemanes habían inventado un procedimiento para hacer más luminosos los instrumentos ópticos. Así cuando al amanecer y próxima la noche, los instrumentos enemigos no detectaban los blancos, ellos acertaban siempre porque podían ver. Estos procedimientos ópticos empezaron a estudiarse, enseguida, en EEUU y en otros países.

En realidad, se trataba de unas láminas antirreflectoras que recubrían las superficies de las lentes y prismas y hacían más transparentes los instrumentos. Otero consiguió, como muestra, una plaquita que en una zona presentaba el reflejo normal y en otra, mucho más transparente, tenía un color azulado. Hoy en día se recubren con esas láminas violeta-azuladas las superficies de vidrio de todos los instrumentos ópticos: cámaras fotográficas, prismáticos, gafas, etc. 
Fue precisamente esta la tarea de Piedad, durante años: el conseguir esas láminas, cosa nada sencilla porque había muy poca bibliografía sobre el particular y tuvieron que hacer muchos ensayos hasta poder conseguirlo. Todo el equipo del LTIEMA que había ido aumentando seguía con interés aquellos trabajos.

Hacia 1945 quedaron bastante desarrollados los procedimientos de preparación: por depósito, en vacío, de una capa de fluoruro, o por ataque con ácido nítrico de la superficie del vidrio. Por fin, se pudo aplicar el tratamiento a los prismáticos que se fabricaban en el LTIEMA.

Otero estaba encantado con el desarrollo de estas investigaciones y encargó a Piedad que elaborara una amplia memoria para presentarla en la Academia de Ciencias y en el año 1946 se le concedió el Primer Premio de la Academia de Ciencias para trabajos de investigación. Este hecho resultó muy sorprendente en los ambientes científicos y periodísticos pues era la única mujer que recibía tan alto reconocimiento.

Recojo a continuación la Memoria del Instituto de Óptica "Daza de Valdés" del curso 1945-1946, la primera en la que aparece Piedad y que testifica que prestó sus servicios en el Instituto desde el primer momento, por su trabajo y méritos.

"Creado este Instituto en el mes de marzo del año actual, cuenta sólo con nueve meses de actividad, durante los cuales ha iniciado su organización como tal y ha empalmado su producción científica con la que venía desarrollando como Sección del Instituto "Alonso de Santa Cruz". Se encuentran organizadas las secciones de: OPTICA FISIOLÓGICA, HISTORIA DE LA ÓPTICA Y FOTOMETRÍA, ÓPTICA FÍSICA, ÓPTICA GEOMÉTRICA Y CÁLCULO DE SISTEMAS Y ESPECTROS ÓPTICOS. [ ...] Está actualmente en organización una Sección de Química Óptica, que abarcará tratamientos de superficies ópticas y depósitos metálicos en cuya materia la Dra. de la Cierva y el becario Sr. Cacho han realizado una meritísima labor, consiguiendo producir primero, e industrializar más tarde, el proceso de obtención de láminas antirreflectoras sobre vidrio. Esta misma sección ha comenzado el estudio de determinados procesos fotoquímicos que interesan a la óptica...La labor cultural del Instituto se ha iniciado con un Curso sobre Óptica Fisiológica, a cargo del director del Instituto D. José Ma Otero, cuya necesidad queda ampliamente justificada con sólo ver la cantidad de público que asiste y el interés con que lo sigue." [13]

En el mismo documento, se incluye un organigrama del Instituto en el que me gustaría destacar la presencia de tres mujeres: Pilar Carro de Cabello (becaria), Piedad de la Cierva y Mํㅡ Teresa Vigón (colaboradoras). Además, hay un listado de los trabajos publicados por los investigadores. El trabajo publicado por Piedad ("Láminas antirreflectoras") destaca al haber obtenido el primer premio del concurso anual de la Academia de las Ciencias. Los trabajos de los otros investigadores fueron publicados, pero ninguno de ellos logró un reconocimiento como el de Piedad.

\section{Los frutos del trabajo en España: viaje a Washington (1948)}

En las Memorias del Instituto de Óptica leemos:

“Durante el año 1948 estuvieron en el extranjero realizando estudios de ampliación, la Dra Vigón, en el Laboratorio del Politécnico de Zürich, la Dra. de La Cierva, el Dr. Barceló y el licenciado Sr. Canga, en el National Bureau of Standards de Washington y los Dres. Ortix y Sánchez del Río, en la Universidad de Roma". [14]

Piedad llega a Washington en junio de 1948 (Fig. 1) para trabajar un año en la Fábrica de vidrio óptico del National Bureau of Standards. Había poca bibliografía sobre este tema y el vidrio óptico se producía en pocos países. Afirma Piedad:

"Allí hice cuanto pude: analicé materias primas, permanecí algún tiempo en cada Sección tratando de seguir cada uno de los procesos de fabricación, dediqué una atención especial a la fabricación de crisoles y a la inspección de defectos del vidrio y seguí cursos complementarios acerca de Diagrama de fases y Examen de sustancias transparentes con microscopio petrográfico" [15]. 

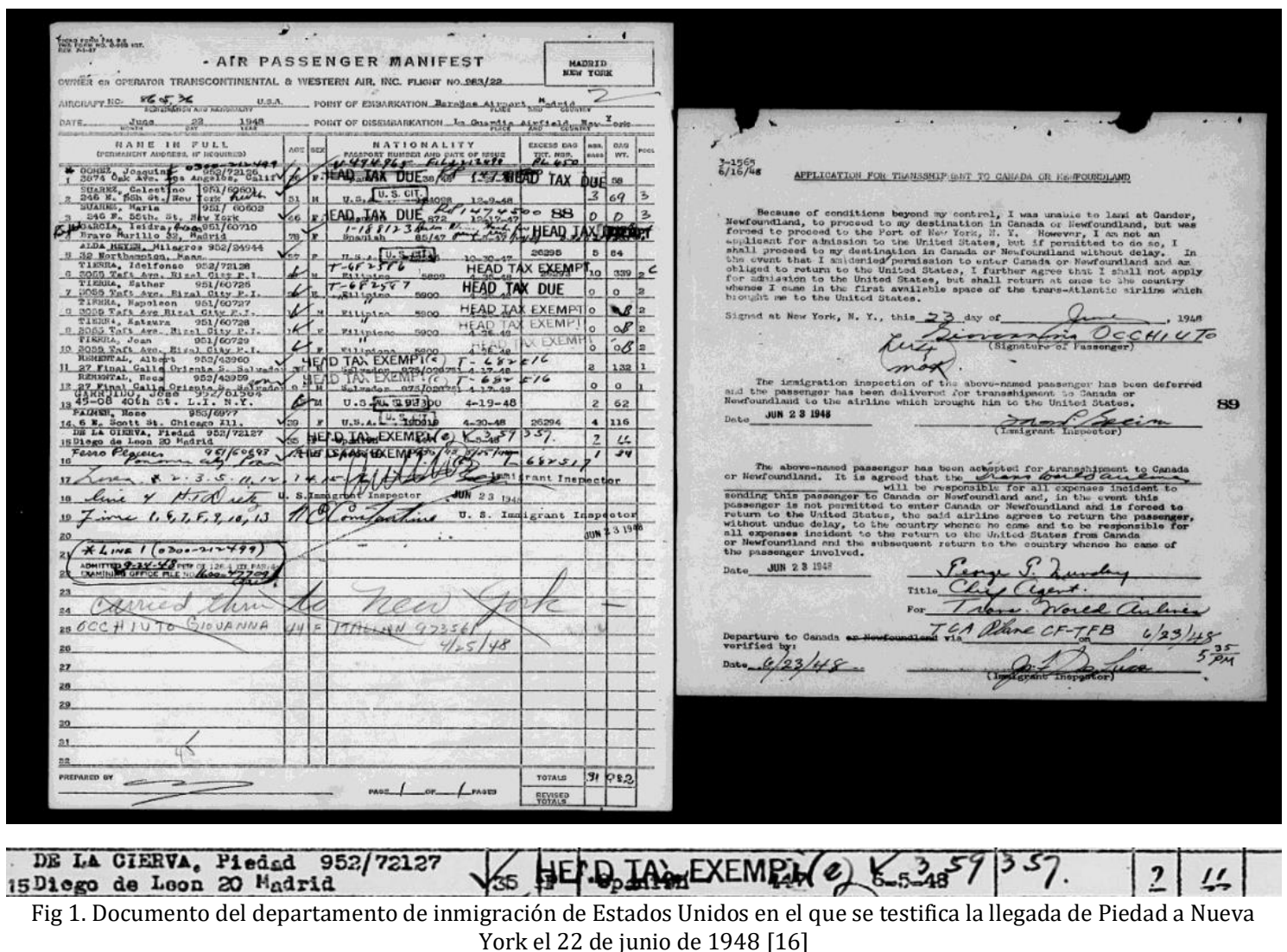

Sucesivamente visitó y estudió en la Facultad de Glass Technology de la Universidad de Toledo y las fábricas Bausch \& Lomb y Kodak en Rochester, N.Y.

Con los conocimientos y la experiencia adquirida, Piedad vuelve a España en 1949 donde:

"No fue fácil comenzar. Reuní el mayor número posible de muestras de materias primas y con el convencimiento de que si se pretende industrializar el país se necesita en primer lugar tipificar nuestros productos naturales, instalé el laboratorio y empecé a analizar. Luego construí el primer horno de ensayos e inicié la fabricación de crisoles pequeños. Era lento y pesado arrancar de la nada...Y continuamos trabajando hasta que, al fin, con pocos medios y mucho cariño, vimos salir de nuestras manos los primeros bloques de vidrio, claros y limpios como diamantes" [15].

El National Bureau of Standards no conserva la estancia de Piedad en su Instituto, aunque sí sabemos cuántos científicos extranjeros trabajaban allí y cómo.

Además, el boletín de 1963 de la American Ceramic Society, relata el trabajo de Piedad de la Cierva en Washington llevado a cabo 15 años antes junto con Luisa Arroyo, que se doctoró en 1962 con una tesis dirigida por Piedad y que trabajó con ella en el LTIEMA [17] (Fig. 2).

Esta sección está en sus inicios, esperando a que terminen de regresar de los E.E.U.U. diversos colaboradores de este Instituto que han estado estudiando problemas relacionados con la fabricación y control del vidrio óptico, los tratamientos del mismo por vía física y química y los distintos tratamientos que pueden interesar aplicar al mismo. En esa Sección se está abordando la fabricación a escala semi-industrial de los tipos más sencillos de vidrio óptico, la determinación de las características físicas y químico-físicas del vidrio etc. Los trabajos publicados por los miembros de la Sección ha sido el siguiente:

P. de la Cierva y Francisco Cacho. “Láminas antirreflectoras” An. Fis. y Quim. Serie A. Física Tomo IV enero-febrero.1949[18].

Hasta 1949 Piedad trabajó en el LTIEMA cuyo objetivo era investigar, desarrollar y producir lentes y aparatos para la Marina con el fin de producirlos industrialmente. Como afirma Ana Romero de Pablos: 
"Tras la segunda guerra mundial la fabricación de vidrio óptico había adquirido gran importancia para los Estados. Era una fabricación costosa y compleja, de interés militar; la óptica fue una pieza importante en gran parte del armamento, que precisó de un fuerte apoyo político. El vidrio óptico se convirtió a partir de entonces en una materia prima fundamental pues fueron muchas las actividades científicas e industriales donde se previó usarlo" [12]

En 1950 Piedad sigue trabajando en el IO incluso como profesora pues en el mismo se realizaban cursos de Óptica Técnica Superior para los alumnos de doctorado.

Piedad de la Cierva and Luisa Arroyo prepared their paper "Study of Optical Glass Surfaces by Leaching with Nitric Acid: I, Polishing Lines" for the June Journat, Dr. de la Cierva, after completing her graduate studies in $\mathrm{X}$ rays and crystal structures, was an assistant professor at the University of Madrid, Spain. During 1948 she visited the United States to observe the Glass and $\mathrm{Re}$ fractories Sections of the National Bureau of Standards. Returning to Spain, Dr, de la Cierva resumed her work at the Laboratorio y Taller de Investigacion del Estado Mayor de la Armada (Navy Research Laboratory), Madrid, where she was research head in the Glass Section when this paper was written. She is now a member of the staff of the Spanish Ceramic Society. Dr. Arroyo graduated in chemistry from the University of Madrid. Since 1951 she has been a research staff member of the Instituto Nacional de Electronica, Madrid. From 1959 to 1963 she also studied the properties of optical glass surfaces under the direction of Dr. de la Cierva at the Laboratorio. This work formed the basis for her Ph.D. dissertation and she received her doctorate in chemistry in 1962.

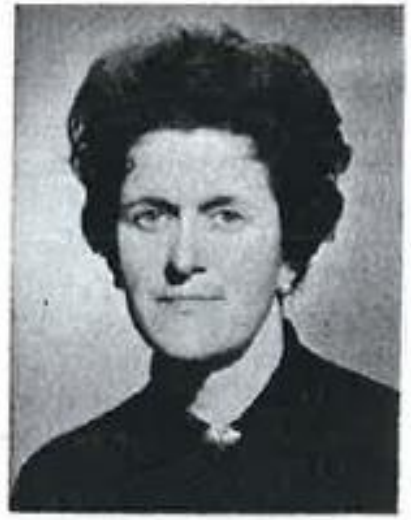

Piedad de la Ciervo

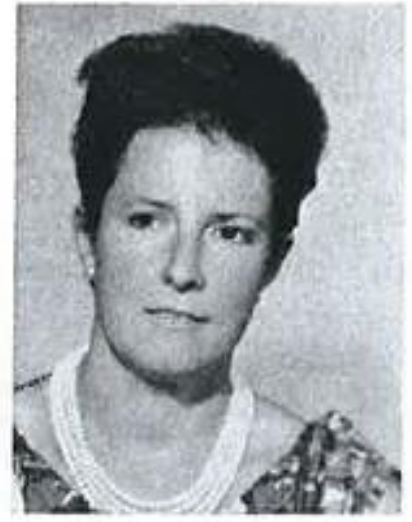

Luisa Arroyo

Fig 2. Boletín de 1963 de la American Ceramic Society en el que se relata el trabajo de Piedad de la Cierva en Washington llevado a cabo 15 años antes junto con Luisa Arroyo.

\section{Las tesis dirigidas por Piedad}

Además del caso de las oposiciones a cátedra de 1940, otro ámbito en el que Piedad sufrió una importante discriminación de género fueron varias tesis que dirigió a diferentes doctorandas.

La primera es la de Francisca de Andrés, colaboradora de Piedad que, en 1949, interesada en las arcillas, trabajó con Piedad de la Cierva su tesis con el título "Estudio físico-químico de arcillas y caolines españoles". Consciente de que la importancia del trabajo en esos años se hubiera podido ver 
afectada por la dirección de una mujer no catedrática, Piedad tuvo que pedirle a José María Albareda, catedrático de geología de la Universidad Complutense de Madrid y futuro rector de la Universidad de Navarra, que apareciera como director.

El segundo caso es de diez años posterior, en 1959, cuando Luisa Arroyo, que había viajado con ella a Estados Unidos y trabajado estrechamente con Piedad, empezó a trabajar acerca de las superficies pulidas de vidrio. Este tema había sido estudiado profundamente por Piedad y Luisa. Ésta, después de licenciarse en Química en la Universidad de Madrid, desde 1951 había sido investigadora en el Instituto Nacional de Electrónica. Desde 1959 empezó a estudiar las propiedades de las superficies del vidrio óptico, bajo la dirección de Piedad de la Cierva. Todo esto formó la base de su tesis que le permitió obtener grado de doctor en 1962. En este caso también otro profesor tuvo que figurar como director.

Seis años más tarde (1965), otra investigadora del LTIEMA, Guadalupe Ortiz de Landázuri, quiso presentar su tesis, también supervisada por Piedad. El trabajo profundizaba en temas relacionados con la capacidad de aislamiento de materias vegetales y obtuvo la máxima calificación cum laude y premio de Investigación "Juan de la Cierva". Como en los dos casos anteriores, Piedad no pudo figurar como directora y esta vez se ofreció el profesor Ángel Viau.

Es por lo tanto destacable el trabajo desarrollado por Piedad, tanto en su línea de investigación como su importancia en impulsar y amplificar el trabajo y la visibilidad de otras mujeres científicas.

Desde 1951 hasta 1976 Piedad siguió trabajando para la Marina como personal civil en el desarrollo de vidrio óptico porque podía tener aplicación inmediata para la fabricación de ladrillos refractarios aislantes, para las calderas de los barcos y para otros hornos como los de cemento que en aquella época de gran impulso de la construcción se importaban de Dinamarca [12]. Desgraciadamente la enfermedad de la madre la obligó a jubilarse a los 63 años.

\section{Conclusiones}

El trabajo de Piedad de la Cierva, su desarrollo personal y académico le hacen merecer el "título" de pionera en las ciencias en una situación histórica muy compleja sobre todo para las mujeres. Por estas razones sorprende que todavía no se haya escrito una biografía completa sobre ella (lo más completo sobre ella puede verse en [11]). Sus aportaciones al mundo de la óptica son muy relevantes, así como su participación en la creación del Instituto de Óptica y su trabajo en el Laboratorio y Taller de Investigación del Estado Mayor de la Armada (LTIEMA). Esperamos poder pronto profundizar sobre este tema accediendo a su archivo personal que se encuentra en Murcia y que todavía no es consultable. 